Rev. Elev. Méd. vét. Pays trop., 26 (4) : 61 a - 69 a

\title{
La synchronisation de l'œstrus chez les ruminants
}

\author{
par J. FERNEY $\left({ }^{*}\right)$ et A. SERE $\left({ }^{*}\right)$
}

\begin{abstract}
RESUME
Les auteurs rappellent les indications générales de la synchronisation de l'cestrus chez les ruminants en soulignant les indications spéciales à l'élevage en zone tropicale: contingences saisonnières, insémination artificielle en élevage extensif, constitution de «feed-lots». Les bases physiologiques du contrôle du cycle cestral, ainsi que les divers stéroïdes anovulatoires utilisables sont ensuite passés en revue.

Pour la synchronisation de l'œstrus chez la vache, il est fait état d'essais pratiqués au Sénégal avec l'acétate de médroxy-progestérone à la dose de $180 \mathrm{mg} /$ jour pendant 18 jours per os (traitement long) et avec la nor-éthandrolone à la dose de $5 \mathrm{mg} /$ jour, couplée avœ une injection de valérianate d'cestradiol au début et une injection de P.M.S. à la fin (traitement court). Ces essais recoupent dans leurs résultats les recherches entreprises ailleurs, mais ils devront être repris sur une plus grande échelle, afin de pouvoir en tirer des conclusions valables.

Chez la brebis, où les indications de la synchronisation de l'cestrus en zone tropicale restent limitées, le procédé des éponges vaginales à l'acétate de fluorogestone constitue la méthode de choix.

En conclusion, l'accent est mis sur la relativité de la méthode qui ne peut réussir que si, par ailleurs, les impératifs classiques de l'élevage sont respectés: sélection et alimentation rationnelle, appliquées à un cheptel sain.
\end{abstract}

La disparité croissante entre les besoins en protéines animales de la population mondiale et les quantités mises à sa disposition fait un devoir aux vétérinaires et aux zootechniciens de promouvoir par tous les moyens les productions animales: viande et lait.

Une plus grande maîtrise des phénomènes de la reproduction, sorte d'eugénisme ne soulevant pas heureusement chez les animaux les mêmes problèmes moraux, sociaux et politiques que dans l'espèce humaine, est un de ceux-ci.

Le but est avant tout d'augmenter le taux de fécondité des animaux, mais aussi de diminuer les contraintes qui sont importantes en matière d'élevage : surveillance des inséminations naturelle ou artificielle, des accouchements, de l'allaitement, rythme d'affouragement, etc.

Depuis une dizaine d'années, la science propose un certain nombre de techniques d'élevage susceptibles d'apporter une solution aux problèmes précédents. Citons d'après ORTAVANT (17) :

- la synchronisation des cycles sexuels dans les différentes espèces domestiques;

- l'induction des naissances gémellaires chez les bovins;

- l'accroissement de la fréquence des mise bas chez les ovins avec comme objectif le double agnelage annuel ou l'agnelage à contre-saison;

$\left(^{*}\right)$ Professeurs à l'Ecole Inter-Etats des Sciences et Médecine Vétérinaires, Dakar, Sénégal. 
- le déplacement dans un sens économiquement favorable de la saison de reproduction, quand elle est unique dans l'année;

- la synchronisation des naissances en provoquant l'accouchement au moment choisi;

- la transplantation ovulaire de femelles à potentialité génétique élevée sur des femellessupports pour assurer la multiplication rapide des lignées d'élite.

Certaines de ces méthodes n'ont pas quitté le domaine expérimental, mais la synchronisation de l'œstrus est entrée dans celui de la pratique. Son emploi en zone tropicale est susceptible d'améliorer les taux de reproduction chez les bovins et les ovins, à condition que l'état sanitaire et la couverture des besoins nutritifs soient satisfaisants. C'est ce qui justifie le présent exposé. Le cas de la chèvre, responsable de tous les péchés en zone aride, sera volontairement laissé de côté, bien que cette espèce puisse aussi bénéficier de cette méthode.

\section{INTERET DE LA SYNCHRONISATION DE L'GESTRUS CHEZ LES RUMINANTS DOMESTIQUES}

Cette technique offre des perspectives de progrès spectaculaires en élevage qui s'expliquent parce que la reproduction se trouve orientée en vue d'une utilisation zootechnique plus facile ou plus rentable du troupeau, car plus rationnelle. Ces avantages sont d'ordre zootechnique général ou d'ordre économique et propres à chaque espèce. Ils ont été exposés en détail dans de nombreuses publications et en particulier dans celle de LE PROVOST et FROGET (11).

\section{Avantages d'ordre zootechnique général}

\section{Amélioration des productions animales}

L'action est à la fois quantitative et qualitative.

a) Quantitative. - Il est possible de raccourcir les périodes improductives en soumettant les femelles à la reproduction dès leur puberté, en déclenchant l'œstrus pendant les périodes normales de repos sexuel, en avançant le premier cestrus post partum et en augmentant le taux des gestations gémellaires. L'œestrus de la femelle de zébu se caractérise par la discrétion de ses symptômes, et la synchronisation de l'œstrus dans un troupeau rend la détection des chaleurs plus aisée (MAULEON et CHUPIN, 15).

b) Qualitative. - La synchronisation de l'œstrus permet la généralisation de l'insémination artificielle dont le pouvoir améliorateur à l'intérieur d'une population donnée n'est plus à démontrer. Les problèmes de stockage et de transport du sperme sont plus facilement résolus, si un grand nombre de femelles sont à inséminer en même temps. En zone tropicale, ces difficultés se révèlent particulièrement aiguës (HANSEL, 10) et la synchronisation de l'œestrus les résout en partie, facilitant entre autres l'emploi du sperme congelé à basse température. Des essais dans ce sens ont été accomplis au C.R.Z. de Dahra, pour étudier les possibilités de diffusion du sperme d'animaux sélectionnés dans des zones où cela n'était pas possible jusqu'ici (DENIS, 6).

\section{Rationalisation des productions animales}

Le choix des époques de fécondation et indirectement de parturition en fonction des impératifs du marché ou des contraintes imposées par la main-d'cuvre n'est pas encore un objectif prioritaire en zone tropicale.

Par contre, le choix des périodes de gestation et de mise bas en fonction des ressources fourragères est de première importance dans des régions où la longueur de la saison sèche soumet chaque année les animaux à une famine relative, hautement préjudiciable au développement des mères et de leurs produits. 


\section{Avantages d'ordre économique}

\section{Bovins}

Chez le bétail à viande, du fait de la reproduction en élevage extensif, l'application pratique de l'insémination artificielle connaît des limites obligées, car la détection précise des chaleurs sur des vaches en liberté reste aléatoire. La synchronisation des chaleurs pallie cet inconvénient, mais en outre les naissances groupées qui en résultent, permettent la constitution de lots homogènes pour l'engraissement. La technique de l'embouche industrielle en « feed-lot » tendant à s'implanter aussi en zone tropicale, cet avantage n'est pas à négliger.

Chez le bétail laitier, des essais de planification des élevages pour une production laitière continue ont donné des résultats encourageants dans les régions à production laitière intensive. Le principe en est simple: sur quatre lots de vaches laitières, il est possible de prévoir quatre époques de vêlages régulièrement réparties au long de l'année et par conséquent une production laitière continue pendant 12 mois. En zone tropicale, les facteurs limitants traditionnels de la production laitière (faible aptitude laitière des races locales, conditions climatiques défavorables, affouragement irrégulier) restreignent dans l'immédiat la portée de cette méthode.

\section{Ovins}

En Afrique tropicale, à la différence des zones tempérées, il n'y a pas de saison de reproduction caractérisée, puisque l'activité génitale de la femelle tend à prendre le type continu et qu'ainsi les agneaux naissent pratiquement toute l'année.

Ainsi la recherche de l'agnelage à contre-saison n'a pas sa raison d'être. Le groupage des chaleurs ne mériterait d'être envisagé que dans l'hypothèse d'un engraissement intensif d'agneaux en «feed-lot» ou lorsqu'on veut profiter de l'augmentation saisonnière des cours, à l'occasion des fêtes traditionnelles en pays islamique (ex. Tabaski). Mais la priorité pour l'instant est réservée en ce domaine à l'engraissement des bovins.

\section{BASES PHYSIOLOGIQUES DE LA SYNCHRONISATION DE L'GSTRUS}

Chez les mammifères à cycle œstrien, la phase lutéale occupe la plus grande partie du cycle et tant que la sécrétion de progestérone par le corps jaune se maintient, le développement folliculaire du cycle suivant est inhibé. La progestérone exerce une rétroaction négative sur l'hypothalamus, à la fois sur le centre de la tonicité en diminuant le niveau de base des hormones gonadotropes et sur le centre de la cyclicité en prévenant l'action des cestrogènes et en empêchant ainsi la décharge ovulante de gonadotropine L.H. (hormone de lutéinisation).

On sait que l'énucléation du corps jaune provoque la reprise de la croissance folliculaire et l'apparition d'un nouveau cycle en quelques jours (LEVASSEUR et THIBAULT, 13). Mais la démonstration initiale de l'inhibition de l'ovulation par la progestérone a été apportée par MAKEPEACE (14) en 1937, chez la lapine. Les études de PINCUS (18) à partir de 1953, permirent d'approfondir le mode d'action et d'envisager des applications pratiques à l'aide de la progestérone, mais surtout des nombreux stéroïdes de synthèse d'action similaire, mis à la disposition des endocrinologistes, sous le nom de progestagènes. Le principe d'emploi de ces molécules se résume ainsi : provoquer à volonté et pendant un temps déterminé l'action inhibitrice de la progestérone du corps jaune sur la sécrétion des gonadotropines et bloquer ainsi durant ce même laps de temps l'ovulation.

Les études d'ECTORS (9), effectuées à l'aide d'un progestagène (médroxy-progestérone) chez la ratte et la truie, montrent que l'action de ce dernier porte directement sur le complexe hypothalamo-hypophysaire par inhibition de la sécrétion de L.H., comme le prouvent l'implantation stéréotaxique du produit au niveau de divers noyaux de l'hypothalamus et l'image histologique de l'hypophyse, caractérisée par l'état de repos des cellules L.H., alors que les cellules à prolactine sont en hyperactivité. 
Il convient toutefois de souligner la différence d'optique qui sépare la recherche de la maîtrise des cycles sexuels chez la femme et chez les animaux domestiques. Dans l'espèce humaine, le contrôle du cycle cestral vise essentiellement le contrôle des naissances en supprimant les périodes physiologiques de fécondité : c'est de la contraception. Dans les espèces animales au contraire, le zootechnicien recherche la simultanéité de l'œstrus sur un nombre déterminé de femelles, afin qu'elles soient fécondées en même temps pour les raisons évoquées ci-dessus : c'est de l'eugénisme.

\section{LES PROGESTAGENES OU STEROIDES ANOVULATOIRES UTILISES EN ZOOTECHINIE}

Actuellement, après les essais initiaux avec la progestérone, seuls sont utilisés les progestagènes, car, à la différence de celle-ci, ils peuvent, pour certains, être utilisés par voie buccale. L'énucléation du corps jaune quant à elle n'est pas réalisable en pratique, pour obtenir la synchronisation de l'œstrus.

Le terme de progestagènes s'applique aux corps qui provoquent la transformation prégravidique de l'endomètre et maintiennent la gravidité chez la femelle castrée (O.M.S., 16). Aussi, d'un point de vue dogmatique, les molécules employées pour obtenir le blocage de l'ovulation, à l'exclusion d'une action proprement progestative, devraient être désignées par le terme de «stéroïdes anovulatoires 》(BERTRAND et DESCHANEL, 3).

Les stéroïdes anovulatoires utilisés chez les mammifères domestiques sont rarement les mêmes que ceux qui sont employés comme anticonceptionnels chez la femme. En effet, l'équilibre endocrinien cestrogène/progestérone n'est pas le même chez les animaux (cycle œstrien à longue phase lutéale) que chez la femme (cycle menstruel à phase lutéale plus courte). Enfin, certains stéroïdes comme l'acétate de fluorogestone, actif par voie vaginale et très commode d'emploi chez la brebis, ne présentent absolument pas le même intérêt chez la femme, où la voie orale est le mode d'administration habituel. De la même façon, mais pour d'autres raisons, cette méthode est d'un emploi difficile chez la vache.

Quelques anovulatoires non stéroïdes ont fait l'objet de recherches. Certains ont donné des résultats décevants (ex. le clomiphène chez la vache), d'autres sont surtout employés chez des non-ruminants (ex. I.C.I. 33828 - Aimax ou méthallibure chez la truie). Aussi nous bornerons-nous à énumérer les principaux stéroïdes anovulatoires utilisés à ce titre chez les animaux.

Ils appartiennent soit au groupe des dérivés de la progestérone, soit au groupe des dérivés de la testostérone.

a) Dérivés de la progestérone (et plus particulièrement de la 17-a-hydroxyprogestérone) :

1. l'acétate de médroxyprogestérone ou M.A.P.,

2. l'acétate de chlormadinone ou C.A.P.,

3. l'acétate de mélengestrol ou M.G.A.,

4. le 16- $\alpha-17$ déhydroxyprogestérone acéto-phénide ou Droxone N.D.,

5. l'acétate de fluorogestone (S.C. 9880) ou F.G.A.

b) Dérivés de la testostérone

Les androgènes ont un effet anovulatoire réduit, mais certains dérivés de la 19-nortestostérone se révèlent remarquables à cet égard chez la vache :

1. la nor-éthandrolone,

2. la nor-éthistérone,

3. le nor-éthynodrel. 


\section{LA SYNCHRONISATION DE L'GSTRUS CHEZ LA VACHE}

Depuis une dizaine d'années, cette méthode a fait l'objet de nombreuses recherches. A défaut de pouvoir les énumérer toutes, il nous suffira de mentionner les équipes responsables des protocoles expérimentaux les plus complets (DERIVAUX et ECTORS, 7; HANSEL, 10; CHUPIN, PETIT et MAULEON, 4).

Technique. - Le tableau suivant donnera une idée de la multiplicité des produits essayés, de leur voie d'absorption et des doses utilisées:

TABLEAU $N^{\bullet}$ I

\begin{tabular}{|l|l|c|}
\hline \multicolumn{1}{|c|}{ Produit } & Voie d'administration & Doses \\
\hline Acétate de médroxyprogestérone & Orsle & $180 \mathrm{mg} / \mathrm{j}$ \\
Acétate de chlormadinone & Orale & $20 \mathrm{mg} / \mathrm{j}$ \\
Acétete de mélengestrol & Orale & $0,5-1 \mathrm{mg} / \mathrm{j}$ \\
Droxone & Orale & $120-150 \mathrm{mg} / \mathrm{j}$ \\
Acétate de fluorogestone & Orale & $20 \mathrm{mg} / \mathrm{j}$ \\
& Intra-musculaire & $2,5 \mathrm{mg} / \mathrm{j}$ \\
& Eponge vaginales & $200 \mathrm{mg}$ \\
Nor-êthístërone & Orale & $0,6 \mathrm{mg} / \mathrm{kg} / \mathrm{j}$ \\
Nor-éthynodre1 & Sous-cutanée & $0,08 \mathrm{mg} / \mathrm{kg} / \mathrm{j}$ \\
& Capsule orale & $0,8 \mathrm{mg} / \mathrm{kg} / \mathrm{j}$ \\
Nor-êthandrolone & Intra-musculaire & $5 \mathrm{mg} / \mathrm{j}$ \\
& Implants sous-cutanés & $250 \mathrm{mg}$ \\
& Eponges vaginales & $800 \mathrm{mg}$ \\
\hline
\end{tabular}

Ces données brutes appellent quelques commentaires :

Le produit. - Il existe de considérables variations de la dose efficace d'un produit à l'autre.

La voie d'administration. - La dose nécessaire est toujours plus élevée, si la voie choisie est orale (10 à 20 fois plus), car il faut une dose qui, tout en n'étant pas prohibitive par son prix, soit suffisante pour tamponner les variations de quantités consommées. extensif.

L'injection journalière présente une lourde sujétion sur les grands effectifs en élevage

L'implant est une solution séduisante, mais il doit être facile à placer et à enlever.

La voie vaginale, sous forme d'éponges, présente certaines difficultés d'emploi chez les bovins, liées à la dimension du vagin et à sa contractilité dans cette espèce.

La durée du traitement. - Il doit se terminer après l'involution du corps jaune progestatif du cycle précédent : sa durée est donc de 18 à 21 jours (traitement long). Il sera plus long lors d'administration orale du fait de l'importance des préestomacs qui rend l'absorption irrégulière. L'administration d'astrogènes (valérianate d'cestradiol, $5 \mathrm{mg}$ ), lutéolytiques chez la vache, au $2^{e}$ jour du traitement, permet de raccourcir la durée de celui-ci à 9-12 jours (traitement court).

En application de ces données, des essais ont été faits au Centre de Recherches Zootechniques de Dahra. Deux protocoles furent mis en cuvre :

a) Traitement long: acétate de médroxyprogestérone à la dose de $180 \mathrm{mg} /$ jour pendant 18 jours per os.

b) Traitement court : nor-éthandrolone à la dose de $5 \mathrm{mg}$ /jour pendant 10 jours par voie 
intramusculaire, avec une injection de valérianate d'œstradiol le $\mathbf{1}^{\text {er }}$ jour pour raccourcir la durée du traitement et une injection de 500 unités de P.M.S. le dernier jour, pour faciliter la maturation folliculaire.

Ces essais encourageants méritent d'être repris sur une plus grande échelle afin de pouvoir en tirer des conclusions valables (DENIS, 6).

\section{Résultats}

$\mathrm{Au}$ terme du traitement, qui a débuté à un moment quelconque du cycle, tous les animaux sont dans un état endocrinien voisin de celui du pro-œstrus d'un cycle œstral normal. Celui-ci s'amorce pour aboutir à l'œstrus et à l'ovulation.

La valeur des résultats s'exprime tout d'abord par le pourcentage d'cestrus induits sur les vaches traitées : (50 à 80 p. 100 en moyenne) et par le pourcentage de fécondation sur les femelles saillies, au premier œstrus induit ou au suivant. Il est possible d'améliorer la maturation folliculaire par une injection de sérum de jument gravide (P.M.S.) à la fin du traitement. Cette technique est particulièrement indiquée chez les génisses prépubères et chez les vaches en anæstrus...

La fertilité est diminuée : 75 à 80 p. 100 des femelles seulement sont fécondées au cours des deux premiers œstrus. Il semble que le "phénomène de rebond », c'est-à-dire la fécondité améliorée, si souvent signalée chez la femme, n'existe pas chez les animaux. A cela plusieurs causes : une migration des spermatozoïdes et une nidation de l'ovule défectueuse, liées l'une et I'autre à une mauvaise préparation endocrinienne du tractus génital femelle. Pour remédier à la pénurie, on préconise classiquement d'inséminer avec un nombre plus élevé de spermatozoïdes.

Il n'en reste pas moins que de nombreux facteurs influent sur les résultats de la méthode, aussi bien sur le pourcentage d'œstrus induits que sur celui des gestations obtenues. Il est bon de les connaître pour utiliser les progestagènes à bon escient :

- les résultats sont moins bons sur les races à viande que les races laitières, sans doute à cause des conditions d'élevage, rendant les contrôles difficiles sur les troupeaux au pâturage;

- les résultats sont meilleurs chez les génisses que chez les vaches adultes.

\section{Inconvénients et dangers}

Ils sont encore mal connus, car tous les expérimentateurs manquent d'un recul suffisant pour les apprécier. Toutefois, sans vouloir ni les minimiser, ni les exagérer, il convient d'en être averti.

L'action anabolisante des dérivés de la 19 nor-testostérone pourrait se révéler gênante pour le fœtus, s'ils sont administrés à des femelles dont l'état de gestation est méconnu.

L'effet œstrogène de certains progestatifs peut diminuer la production laitière. Aussi sont à exclure de l'usage zootechnique sur le bétail laitier les anticonceptionnels (ex. nor-éthinodrel) utilisés chez la femme et a fortiori les préparations contenant un œstrogène et un progestatif, indiquées pour des raisons différentes chez la femme.

Enfin se pose le problème des métabolites résiduels éliminés par le lait et susceptibles d'interférer sur le développement génital du jeune à l'allaitement.

Le dossier n'est donc pas clos et de nouvelles observations sont encore nécessaires pour améliorer la méthode et vérifier son innocuité totale.

\section{LA SYNCHRONISATION DE L'ESTRUS CHEZ LA BREBIS}

Le principe en est le même chez la vache. Mais la physiologie sexuelle propre à la brebis oblige, en zone tempérée, à envisager le problème avec des possibilités différentes. 
Avec BADINAND (1) on est amené, en zone tempérée, à rechercher le déclenchement et la synchronisation de l'œstrus

- soit chez les adultes

- pendant la saison sexuelle

- quelques semaines avant cette période

- pendant l'anœstrus séparant deux saisons sexuelles

- soit chez les jeunes en contre-saison ou au cours de la saison sexuelle.

En zone tropicale, où la saison de reproduction dure pratiquement toute l'année, et où la mise précoce à la reproduction des agnelles n'est peut-être pas souhaitable, compte tenu des conditions d'élevage, la seule indication rationnelle semble être le groupage des chaleurs de brebis, en période de reproduction, pour obtenir des lots d'agneaux de même âge pour l'engraissement. Ceci suppose déjà un effectif important au départ pour que l'opération soit rentable. Cette vue est sans doute encore fort prospective et c'est pourquoi nous n'exposerons, des procédés utilisables, que leurs grandes lignes.

Technique. - A quelques différences près, les produits employés chez la vache sont utilisables chez la brebis.

La progestérone. - Elle a été à la base des recherches sur la synchronisation de l'œstrus. Depuis 1948, les travaux de DUTT et CASIDA (8), de DAUZIER et collab. (5), et de ROBINSON (20) ont montré que l'injection journalière de progestérone pendant 2 à 3 semaines à des brebis au cours de la saison de reproduction inhibait l'œstrus, qui réapparaissait 2 à 5 jours après la fin du traitement.

Les progestagènes. - Trois seulement, parmi les nombreux corps similaires de la gamme, se partagent les faveurs des praticiens chez la brebis. Ils sont utilisés selon les techniques suivantes:

TABLEAU $\mathrm{N}^{\circ} \mathrm{II}$

\begin{tabular}{|l|l|c|c|}
\hline \multicolumn{1}{|c|}{ Produits } & Voie d'administration & Dose journalière & Durée du traitement \\
\hline Acétate de médroxyprogestërone (MAP) & Orale & $50-75 \mathrm{mg}$ & $14 \mathrm{j}$ \\
Acétate de chlormadinone (CAP) & Orale & $1-2 \mathrm{mg}$ & $18 \mathrm{j}$ \\
Acétate de fluorogestone (SC.9880) & Eponges vaginales & $30 \mathrm{mg}$ & $17 \mathrm{j}$ \\
\hline
\end{tabular}

Alors que chez la vache, la voie vaginale n'offre que peu d'intérêt, chez la brebis cette méthode se révèle le procédé de choix. C'est à ROBINSON (20) que revient le mérite d'avoir mis au point le procédé : une éponge de polyuréthane, imprégnée de $30 \mathrm{mg}$ d'acétate de fluorogestone est laissée en place pendant 17 jours. On admet qu'une telle éponge libère environ $80 \mathrm{p}$. $100 \mathrm{du}$ produit par jour, soit $2,4 \mathrm{mg}$ le premier jour et $0,7 \mathrm{mg}$ le $16^{\mathrm{e}}$ jour.

\section{Résultats}

Pour les progestatifs administrés par voie orale à des brebis au cours de la saison de reproduction, le pourcentage d'œstrus induit est de 77 à 85 p. 100 avec une fécondation de l'ordre de 37 à 56 p. 100 . Les meilleurs taux sont obtenus avec le M.A.P.

Avec l'acétate de fluorogestone en éponges vaginales, 96 p. 100 des brebis présentent un cestrus entre la $36^{\circ}$ et la $72^{\mathrm{e}}$ heures qui suivent le retrait de l'éponge, avec une fécondité de $55 \mathrm{p}$. 100 environ.

La fécondité au deuxième astrus qui survient 16 à 17 jours plus tard est supérieure à la normale (BADINAND et LAGNEAU, 2). Sur les brebis en période d'activité sexuelle, l'injection en fin de traitement de P.M.S. n'apporte pas d'amélioration sensible. Il semble que la baisse de 
fécondité soit la conséquence d'une migration difficile des spermatozoïdes qui sont retrouvés dans l'oviducte en nombre dix fois moins élevé sur les femelles traitées que sur les femelles inséminées pendant un œstrus normal.

Le champ d'application des progestagènes offre de réelles possibilités pour maîtriser le cycle reproducteur de la brebis. L'agnelage à contre-saison en particulier est une des meilleures indications des progestagènes en zone tempérée. Aussi leur emploi en zone tropicale reste-t-il pour l'instant d'un intérêt limité. D'autant plus que ces méthodes d'élevage réclament pour réussir des troupeaux d'effectifs suffisants et en parfait état, aussi bien sanitaire que nutritionnel. Une expérience de cet ordre tentée au Sénégal a tourné court, à cause de l'état sanitaire du troupeau.

Au terme de cet exposé, le bilan de l'emploi des progestagènes pour obtenir la synchronisation de l'œstrus sur les bovins et les ovins tropicaux peut paraître décevant. En effet, il est inutile de demander à la synchronisation de l'œstrus plus que la valorisation d'une production animale déjà d'un niveau supérieur à la moyenne. Vouloir en faire un palliatif des imperfections ou des insuffisances d'un élevage, conduit inévitablement à de graves mécomptes. On ne fait pas de l'élevage avec un médicament et une seringue, mais en obéissant à des lois qui, malgré les améliorations apportées aux techniques par l'évolution de la science, restent dominées par la sélection et l'alimentation rationnelle, appliquées à un cheptel sain. L'augmentation des productions animales repose avant tout sur l'amélioration de l'état sanitaire par une prophylaxie raisonnée et suivie des maladies infectieuses et parasitaires et celle de l'état de nutrition par la limitation des contraintes alimentaires imposées par le climat.

Les lignées d'animaux existantes pourront ainsi extérioriser au maximum leurs potentialités génétiques, qui sont grandes, puisqu'elles survivent et produisent malgré tout. La synchronisation de l'œstrus, comme les autres techniques de pointe énoncées au début, pourront alors intervenir avec leur plein effet.

\section{SUMMARY}

\section{The synchronization of oestrus applied to ruminants}

The authors bring back to mind the general indications of the synchronization of oestrus applied to Ruminants, emphasizing such indications as are peculiar to cattle and sheep breeding in the tropical zone: namely seasonal contingencies, artificial insemination in extensive breeding, and the establishment of feed-lots. The physiological basis of the control of the ovarian cycle, together with the various anovulatory steroids which can be used, are then considered.

As for the synchronization of oestrus applied to cows, the authors bring to attention some practical experiments conducted in Senegal with Medroxy-progesterone acetate, at the rate of 18 daily doses of $180 \mathrm{mg}$ per os (protracted treatment), and with nor-ethandrolone at a $5 \mathrm{mg}$ daily dose in association with one injection of oestradiol valerianate at the beginning and one injection of P.M.S. at the end (short treatment). In their results, these experiments coincide with those undertaken by other research workers, but they will have to be re-conducted on a larger scale, so that valid conclusions may be reached.

Concerning ewes, as the indications for the synchronization of oestrus in the tropical zone remain limited, the use of fluorogestone impregnated intravaginal sponges appears as the best method.

The authors conclude by emphasizing the relativity of such a method which can only succeed if, at the same time, traditional breeding imperatives are respected: selection and rational feeding as applied to healthy livestock. 


\section{RESUMEN}

\section{La sincronización del estrus en los rumiantes}

Los autores recuerdan las indicaciones generales de sincronización del estrus en los rumiantes y particularmente en lo concerniente a la cria tropical: contingencias estacionales, inseminación artificial en cria extensiva, instalación de \& feed-lots $"$. Pasan en revista las bases fisiologicas del control del ciclo estral asi como los varios steroidos anovuladores utilizables.

Para la sincronización del estrus en la vaca, se hicieron ensayos prácticos en Senegal con el acetato de medroxy-progesterona en dosis de $180 \mathrm{mg} /$ dia durante 18 dias per os (tratamiento largo) y con la norethandrolone en dosis de $5 \mathrm{mg} / \mathrm{dia}$, junta con una inyección de valerianato de estradiol al principio y una inyección de P.M.S. al fin (tratamiento corto). Los resultados de estos ensayos coinciden con investigaciones hechas en otra parte. Dichos ensayos necesitaran ser perseguidos en gran escala para conseguir conclusiones valederas.

En la oveja en la cual las indicaciones de la sincronisación del estrus en region tropical quedan limitadas, el proceder de las esponjas vaginales con acetato de fluorogestone constituye el método de primera calidad.

En conclusión, los autores recalcan la relatividad del método que puede tener exito sólo si, por lo demás, se respetan los imperativos clásicos de la cria : selección y alimentación racional, aplicadas en ganada sano.

\section{BIBLIOGRAPHIE}

1. BADINAND (F.). Synchronisation des chaleurs chez les petits ruminants. Econ. Méd. anim., 1970 , 11 (6) : 333-346.

2. BADINAND (F.) et LAGNEAU (F.). Modification artificielle du cycle sexuel chez les petits ruminants. I. Synchronisation des chaleurs chez la brebis. Rec. Méd. Vét., 1969, 145 (10) : 1041-1053.

3. BERTRAND (M.) et DESCHANEL (J.P.). Le contrôle des cycles sexuels chez la vache et la chienne. Econ. Méd. anim., 1970, 11 (6) : 319-333.

4. CHUPIN (D.), PETIT (M.) et MAULEON (P.). Maîtrise de l'cestrus et synchronisation des cycles sexuels chez les bovins. Bull. Techn. Inf. Minist. Agric., 1971 (257) : 163-174.

5. DAUZIER (L.), ORTAVANT (R.), THIBAULT (C.) et WINTERBERGER (S.). Recherches expérimentales sur le rôle de la progestérone dans le cycle sexuel de la brebis et de la chèvre. Ann. Endocrin., $1953,4: 553-559$.

6. DENIS (J. P.). Communication personnelle.

7. DERIVAUX (J.) et ECTORS (F.). Aperçus sur les progestagènes et la synchronisation de l'astrus. Cah. Méd, vét., 1966, 35 (2) : 63-89.

8. DUTT (R.H.) et CASIDA (L.E.). Alteration of the estrual cycle in sheep by use of progesterone and its effects upon subsequent ovulation and fertility. Endocrinology, 1948, 43 : 208-217.

9. ECTORS (F.). L'étude histo-pathologique du mécanisme d'action de la médroxy-progestérone. 1 vol., Bruxelles, Arscia, 1971, 175 p.

10. HANSEL (W.). Control of the ovarian cycle in cattle. In : LAMMING (G. E.) et AMOROSO (E. C.). Reproduction in the female mammal. London, Butterworth, 1967.

11. LE PROVOST (F.) et FROGET (J.). Motivations zootechniques générales et intérêt économique de l'utilisation des progestagènes chez les femelles domestiques. Econ. Méd. anim., 1971, 12 (1): 5-19.

12. LE PROVOST (F.), THIMONNIER (J.) et MAULEON (P.). Obtention de gestation à contre-saison à l'aide d'éponges vaginales imprégnées d'acétate de fluorogestone (S.C. 9880) chez différentes races françaises de brebis. VIe Cong. Reprod. Insém. Artif., Paris, 1968.

13. LEVASSEUR (M. C.) et THIBAULT (Ch.). Bases physiologiques de l'utilisation des progestagènes chez les mammifères domestiques. Econ. Méd. anim., 1971, 11 (6) : 311-317.

14. MAKEPEACE (A. W.), WEINSTEIN (G. L.) et FRIEDMAN (M. H.). Am. J. Physiol., 1937,119 : 512.

15. MAULEON (P.) et CHUPIN (D.). Maîtrise des cycles sexuels chez les bovins. Econ. Méd. anim., 1971, $12(1): 31-44$.

16. Organisation Mondiale de la Santé. Aspects cliniques de lutilisation des gestagènes oraux. Genève, 1966. (Rapport technique $\mathrm{n}^{\circ} 326$ ).

17. ORTAVANT (R.). Reproduction des animaux domestiques. Cah. Ing. Agron., 1970, (251): 17-20.

18. PINCUS (G.). The control of fertility. New York, Academic Press, 1965.

19. PINCUS (G.) et CHANG (M. C.). Acta Physiol. Latinoon, 1953, $3: 177$.

20. ROBINSON (T. J.). The Control of the ovarian cycle in the Sheep. 1 vol. Sydney, Sydney University Press, 1967, 258 p. 